\title{
Stability diagrams for a memristor oscillator
}

\author{
Jason A.C. Gallas ${ }^{1,2,3, a}$ \\ ${ }^{1}$ Max-Planck-Institut für Physik komplexer Systeme, 01187 Dresden, Germany \\ ${ }^{2}$ Complexity Sciences Center, 9225 Collins Avenue Suite 1208, Surfside, FL 33154, USA \\ ${ }^{3}$ Instituto de Altos Estudos, Rua Silvino Lopes 419-2502, 58039-190 João Pessoa, Brazil
}

Received 23 January 2019 / Received in final form 8 March 2019

Published online 14 October 2019

\begin{abstract}
The simplest chaotic circuit containing a memristor involves a capacitor $C$, an inductor $L$, and two parameters, $\alpha$ and $\beta$, characterizing the memristor. Chaos was observed experimentally for three combinations of these parameters. Here, we report high resolution stability diagrams displaying an abundance of tunable ranges of periodic and chaotic self-oscillations for this circuit, in all six possible control planes. We predict dynamically rich and intricate sequences of oscillations that are experimentally accessible in the system.
\end{abstract}

\section{Introduction}

The goal of this paper is to report a detailed investigation of the tunable ranges that support periodic and complex oscillations in the "simplest chaotic circuit", introduced by Muthuswamy and Chua [1], an autonomous circuit containing two passive linear elements, an inductor and a capacitor, connected in series with a memristor, a nonlinear active element (see Fig. 1). The class of memory circuit elements is interesting because such elements display complex phenomena and are potentially applicable in many fields, ranging from complex circuitry to neurobiology [2-4]. For instance, niobium dioxide Mott memristors were incorporated recently into a relaxation oscillator and found to display a tunable range of periodic and chaotic self-oscillations and to be potentially useful in certain types of neural-inspired computation by introducing a pseudo-random signal that prevents global synchronization and could also assist in finding a global minimum during constrained searches [5,6]. A number of other exciting applications exist, e.g. references [7-11] and references therein.

Muthuswamy and Chua [1] stated that their simple memristor circuit is related to Rössler's circuit, a statement reiterated in subsequent work [12]. A profusion of elusive and complex global phenomena were recently observed in Rössler-like circuits, namely periodicity hubs and infinite nestings of complex stability spirals [13-15]. The importance of periodicity hubs lies in the fact that they are focal points where occurs an accumulation of an infinite quantity of spirals characterized by periodic oscillations of distinct waveforms and periods. Separating each pair of periodicity spirals one finds a spiral of chaos, making the whole set a doubly infinite nested set of spirals forming a kind of "sandwich" of spirals. In other words, periodicity hubs imply a doubly infinite alternation between spirals of periodicity and spirals

${ }^{a}$ e-mail: jgallas@pks.mpg.de 


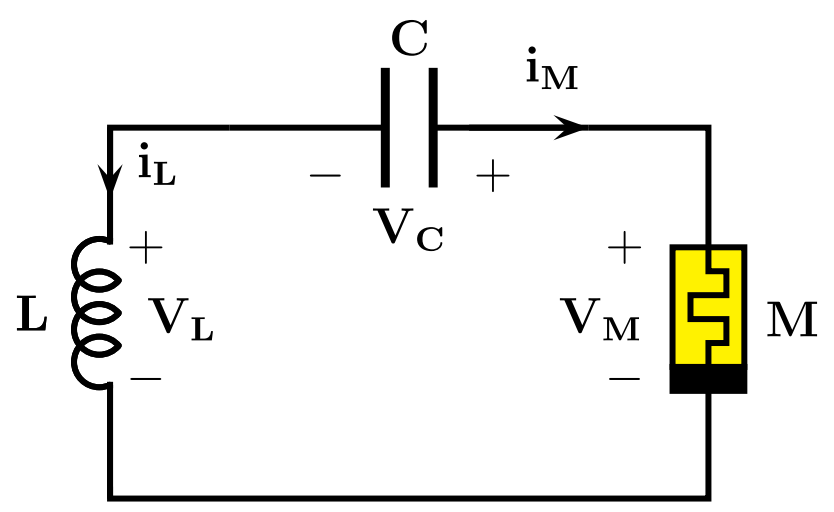

Fig. 1. Schematic circuit considered here, governed by equation (1). Details of the nonlinear memristive element $M$, controlled by parameters $\alpha$ and $\beta$ in equation (1), are discussed by Muthuswamy and Chua [1].

of chaos. Thus, it is possible to move towards the focal hub along any such spirals (chaotic or not), while always remaining confined inside the same spiral (chaotic or not). At the focal hub, it becomes possible to move on to any one of the doubly infinite set of spirals and, from there, to spiral out. During the last decade, such periodicity hubs were found to exist abundantly in the control parameter space of nonlinear dynamical systems [13-15]. For a survey see, e.g., reference [16]. Thus, we were motivated to check whether or not the memristive oscillator would also display elusive characteristics in its control parameter space, characteristics which are known to exist in the standard diode of Chua, either in a cubic or in the piecewise-linear configuration [17]. A secondary reason is that, originally, chaotic oscillations were reported for just three experimental configurations. Normally, for this type of system, chaotic oscillations appear over extended parameter intervals. Therefore, considering the scarcity of the chaotic oscillations, we were also motivated to look for the presence of chaos in the system. In this paper we report our findings.

The dynamics of the memristive oscillator in Figure 1 is governed by three autonomous equations and four parameters [1]:

$$
\frac{d x}{d t}=\frac{y}{C}, \quad \frac{d y}{d t}=-\frac{1}{L}\left[x+\beta\left(z^{2}-1\right) y\right], \quad \frac{d z}{d t}=-y-\alpha z+y z .
$$

As described in detail by Muthuswamy and Chua [1], $x$ and $y$ are proportional to $V_{C}$ and $i_{L}$, respectively, while $z$ describes the internal state of the memristive device. Muthuswamy and Chua [1] fixed

$$
C=1, \quad L=3, \quad \text { and } \quad \alpha=0.6
$$

By varying $\beta$, they reported observing chaotic attractors for $\beta=1.5$ and 1.7 , while period-one and period-two oscillations were observed for $\beta=1.2$ and $\beta=1.3$, respectively.

Numerically, subsequent work found chaos in a circuit closely related to the system above [12]. Chaos and some analytical results were reported for specific choices of $\alpha[12,18,19]$. A number of experimental and numerical results were reported recently for circuits containing mem-components, e.g. [20-24]. However, a systematic investigation of the control parameter space of such systems seems to be still lacking.

Here, high-resolution stability phase diagrams are reported for the six possible control parameter planes of the simplest memristor circuit. The above specific parameter choices were not discussed in the original work. Thus, we decided to investigate 

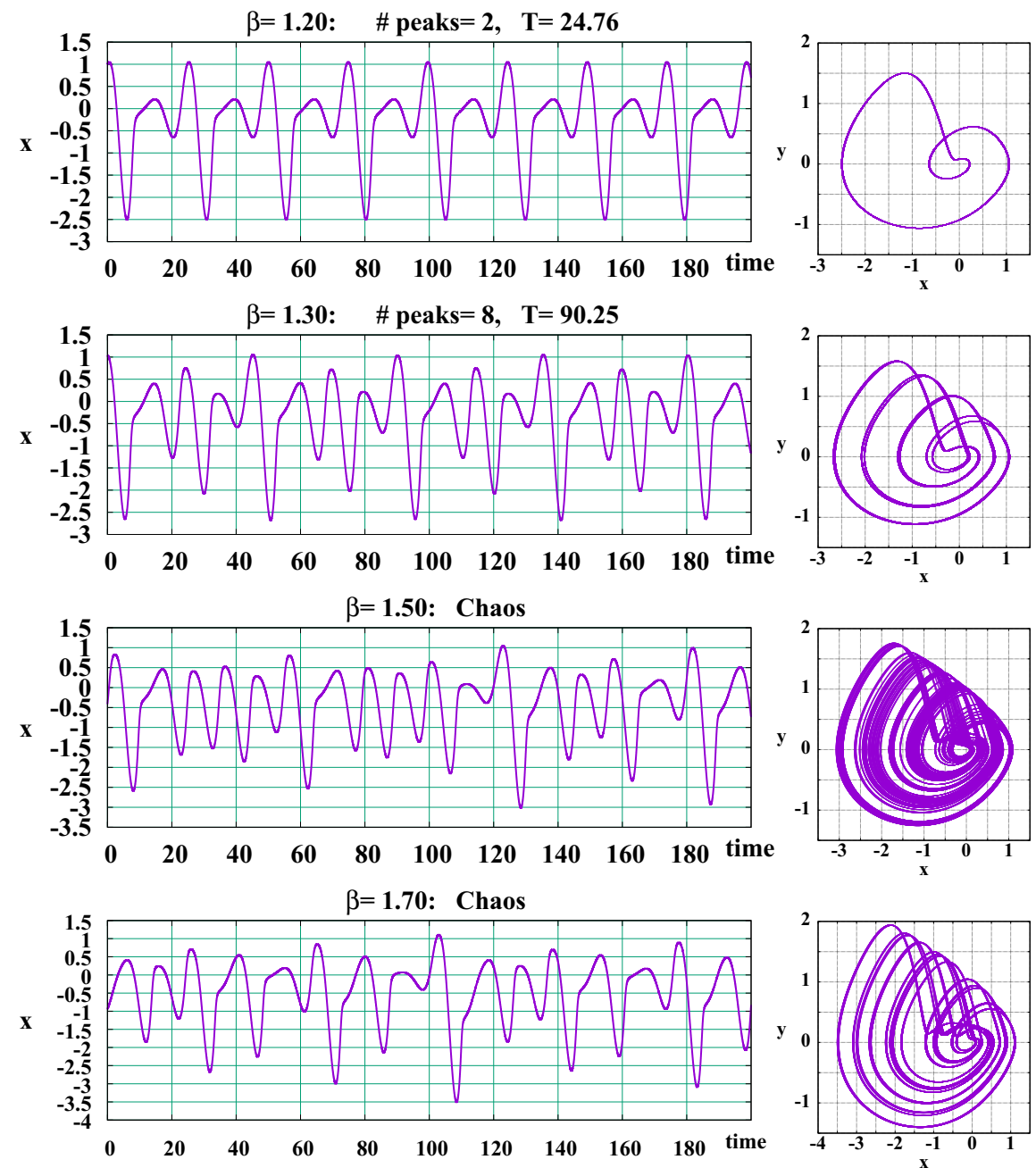

Fig. 2. Temporal evolution of the voltage $x$ and attractor projections for $\beta=1.2,1.3$, 1.5, 1.7. The ratio of the periods $T$ of the non-chaotic signals is $90.25 / 24.76 \simeq 3.64$. The corresponding ratio of the number of peaks (local maxima) per period is 4 . Notice differences in scales: amplitudes increase with $\beta$.

what sort of behaviors exist when parameters are changed, not necessarily by small amounts. The stability diagrams presented below are centered on the parameters originally considered by Muthuswamy and Chua. Remarkably, our stability diagrams predict the existence of very extended tunable ranges of periodic and chaotic selfoscillations for the memristive circuit.

\section{Phase space analysis}

We start by considering standard diagrams involving explicitly the dynamical variables. This is done both to get a preliminary idea of the circuit dynamics, and to compare our numerical computations with earlier findings.

Figure 2 illustrates for $\beta=1.2,1.3,1.5$ and 1.7, the temporal evolution of the voltage $V_{C}$, proportional to $x$ variable, and $y \times x$ projections of the system attractor. 
Following the original work [1], we use the initial conditions $x(0)=0.1, y(0)=0$, and $z(0)=0.1$. Attractors were obtained using a standard fourth-order Runge-Kutta integrator with fixed step $h=0.05$. Using $h=0.005$ produces no visual changes in the graphs. Muthuswamy and Chua found that the attractors for $\beta=1.2$ and 1.3 correspond to their experimentally measured period-one and period-two oscillations, without elaborating on it. In contrast, Figure 2 shows distinct motions for this pair of parameters. While for $\beta=1.5$ we obtain a plot similar to the one in their Figure 2, the panels for $\beta=1.2$ and 1.3 are markedly different.

The $x \times y$ projections in Figure 2 seem to show only periodic, not chaotic attractors. However, the temporal evolution of the voltage $x$, together with the corresponding Lyapunov spectrum (not shown here), unambiguously show that the oscillations are chaotic. Individual panels also record the period $T$ for the periodic oscillations, as well as their number of peaks (local maxima) per period. In Figure 2, "chaos" means a positive Lyapunov exponent. From Figure 2 it is possible to recognize that the peaks of chaotic oscillations tend to repeat with roughly the same amplitude. In other words, local maxima of the oscillations are restricted to very narrow intervals, explaining why projections in Figure 2 give the (incorrect) impression of representing periodic oscillations. Projections of larger portions of the attractor (not shown here) are seen to significantly change general appearance of the projections which, however, continue to give the impression of representing periodic attractors. Although positive, the Lyapunov exponents for $\beta=1.5$ and 1.7 are rather small.

Are there period-one and period-two oscillations located near $\beta=1.2$ and 1.3 ? To check this possibility, we computed the bifurcation diagrams shown in Figure 3, covering the extended interval $0.9 \leq \beta \leq 2$, discretized into a mesh of 800 equidistant points. These bifurcation diagrams were computed in two independent ways: (i) by starting computations always from the same initial condition $x(0)=0.1, y(0)=0$, $z(0)=0.1$ for all points of the mesh; (ii) using the standard technique of "following the attractor", namely starting on the left boundary of the mesh at the initial condition above and, when increasing $\beta$, not re-starting the initial conditions but, instead, using those values of $x, y, z$ that were stored in the computer buffer, from the previous computation. Visually, both sets of diagrams look identical, implying that it is unlikely to find multistability in the parameter regions surveyed. To further check for multistability, we also tried using a few initial conditions taken randomly from the interval $[-1,1]$, with identical outcome. Thus, no period-one and period-two oscillations seem to exist near to $\beta=1.2$ and 1.3. Notice that the narrow intervals of chaotic oscillations at $\beta=1.7$ are clearly corroborated by Figure 3c.

\section{Stability phase diagrams}

As mentioned, the memristor circuit governed by Equation (1) depends on four parameters. Accordingly, we explore all six possible two-dimensional stability diagrams, sections of the control parameter space, centered around the reference point $(C, L, \alpha, \beta)=(1,3,0.6,1.5)$.

Figure 4 shows Lyapunov stability diagrams for the $\beta \times C$ and $\beta \times L$ control planes. All diagrams reported in this paper were computed using standard procedures, as described in detail in, e.g. references [14,16,25]. In Figure 4, colors represent chaotic oscillations (positive Lyapunov exponents) while dark shadings denote parameters leading to periodic oscillations (negative exponents), as indicated by the colorbars. Dots mark the points $\beta=1.2,1.3,1.5,1.7$. Horizontal lines mark either $C=1$ or $L=3$. In Figure 4 and in all Lyapunov diagrams below, the color coding was always renormalized according to the minimum and maximum values of the 



Fig. 3. Bifurcation diagrams showing maxima of $x$ along the line $C=1$. (a) The four vertical lines mark $\beta=1.2,1.3,1.5$ and 1.7. (b) Magnification around $\beta=1.5$. (c) Magnification around $\beta=1.7$, illustrating the very limited variation intervals of the chaotic attractor.

exponents contained by the windows. That is the reason why, for instance, Figure 4b displays a different pattern of colors than the region inside the white box in Figure 4a.

From Figure 4 one recognizes that the Lyapunov diagrams predict the existence of extended tunable ranges of periodic and chaotic self-oscillations. The larger parameter regions in Figures $4 \mathrm{a}$ and $4 \mathrm{c}$ clearly show that regions of periodic motions are far larger than regions of chaos. Furthermore, from Figures $4 \mathrm{~b}$ and $4 \mathrm{~d}$, one recognizes that it is remarkable that Muthuswamy and Chua found two instances of periodic oscillations in a region where the probability of finding chaos is relatively high.

Figure 5 presents Lyapunov stability diagrams illustrating details in successive magnifications of the control region inside the white box in Figure 4d. This figure shows chaos to be dominant in this region. Figure 5 also shows a profusion of shrimps [26-28] which, however, appear stretched in several distinct shapes and configurations 

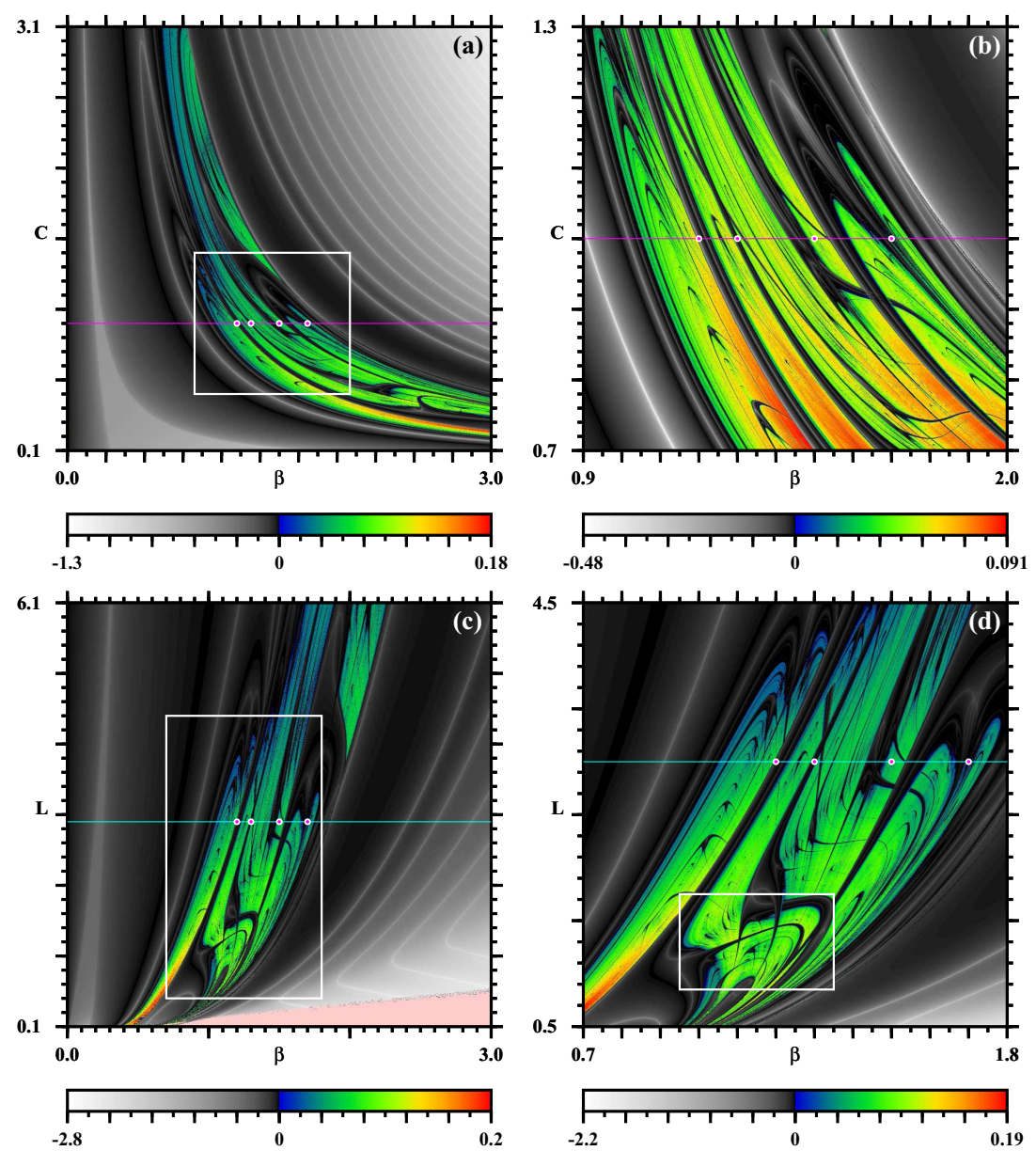

Fig. 4. Lyapunov stability diagrams displaying extended tunable ranges of periodic and chaotic self-oscillations in the $\beta \times C$ and $\beta \times L$ control planes. The white box in the left panels are shown magnified on the right; the white box in $d$ is magnified in Figure 6. Colors represent chaotic oscillations while dark shadings denote periodic oscillations, as indicated by the scale. Dots mark the points $\beta=1.2,1.3,1.5,1.7$. Horizontal lines mark $C=1$ or $L=3$. Individual panels display $1400 \times 1400$ exponents. In c, the pink region at the bottom refers to parameters leading to unbounded attractors (divergence).

but, apparently, do not display signs of periodicity hubs [13] and never coiling up to form infinite spirals [14] or other intricate shrimp networks [29-31]. Of course, since the diagrams presented here reflect the parameter space configuration around the reference point $(C, L, \alpha, \beta)=(1,3,0.6,1.5)$, it is still possible that the aforementioned complex structural self-organizations show up in other parameter regions. The investigation of this interesting problem requires considerable investment of computer-time and is not pursued here.

The leftmost column in Figure 6 presents Lyapunov diagrams showing successive magnifications of the predicted distribution of periodic and chaotic oscillations in the memristor $\beta \times \alpha$ control plane. The stability phases recorded in these diagrams display characteristics similar to the ones already described above. In fact, it is quite remarkable that stability diagrams seem to show quite similar dependences on the linear $(C$ and $L)$ and nonlinear $(\alpha$ and $\beta)$ control parameters. 

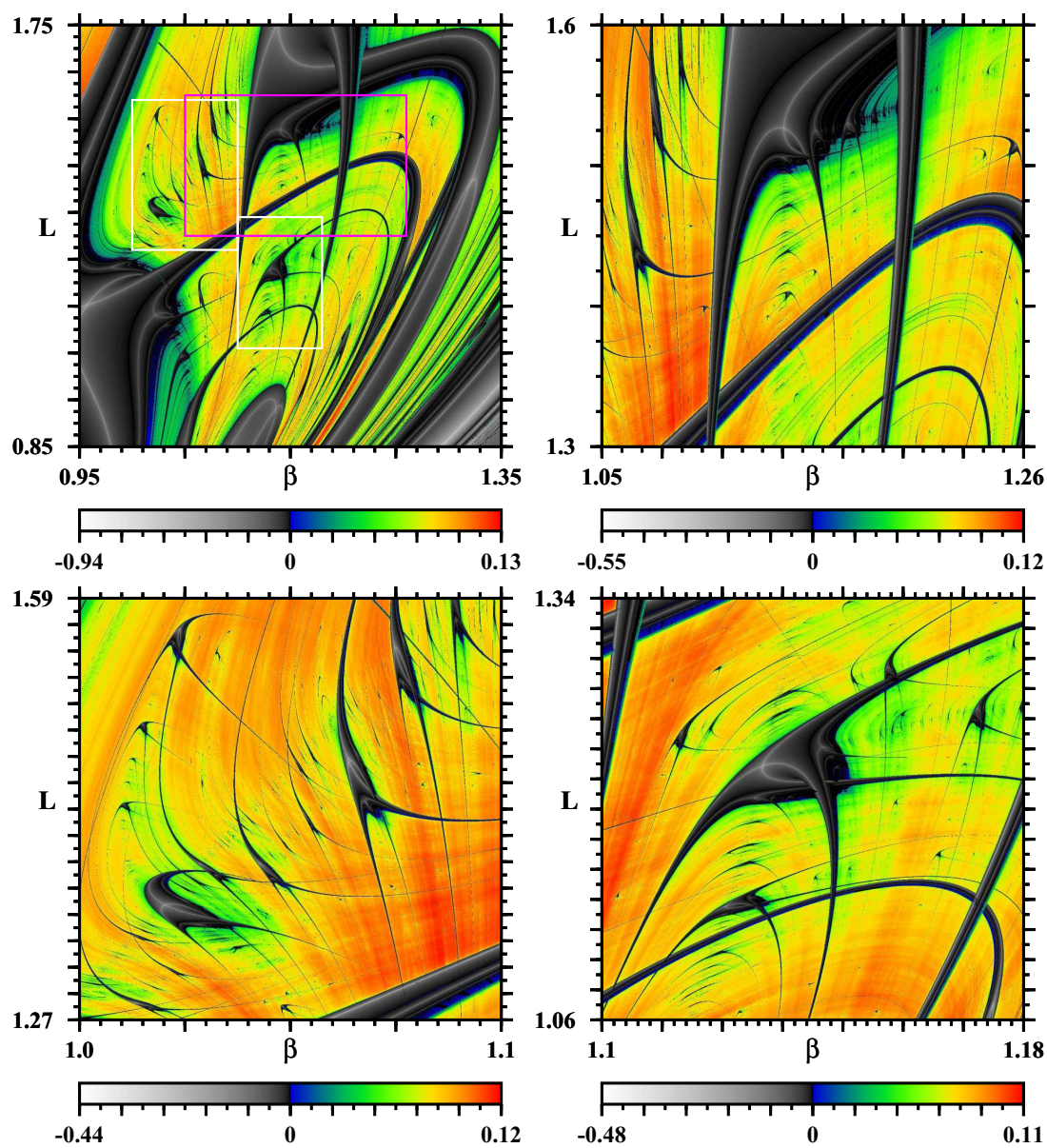

Fig. 5. Successive magnifications illustrating details of the organization of windows of periodic and chaotic self-oscillations in the $\beta \times L$ control plane. Shrimps bend and get stretched, but do not seem to form spirals (see text). Each individual panel displays $1400 \times$ 1400 exponents.

The three rightmost columns in Figure 6 display a complementary type of stability diagrams that we call isospike diagrams [25,32], namely diagrams obtained by counting the number of spikes per period for all periodic oscillations. Isospike diagrams are more informative than Lyapunov diagrams because they not only discriminate periodicity from chaos but, simultaneously, indicate precisely where the number of spikes per period changes when tuning parameters. In particular, the isospike diagrams obtained by counting spikes per period of the $x$ and $z$ variables show considerable similarity when compared to the corresponding ones obtained by counting spikes of $y$. Moreover, as seen from the figures, they expose a lot of intricate substructure in regions where Lyapunov diagrams display no variation.

To compute isospike diagrams, subsequently to the computation of the Lyapunov exponents, integrations were continued for $40 \times 10^{5}$ additional time-steps when up to 800 extrema (maxima and minima) of the variable of interest were recorded, along with the time of their occurrence. A simple search of this series of extrema allowed verifying whether spikes repeated or not. All computations were done in double precision (8-byte real numbers meaning about 15 digits of accuracy). A palette of 19 colors is used to represent the number of spikes per period. Patterns having 



Fig. 6. Tunable stability regions as a function of the memristor parameters $\alpha$ and $\beta$, when fixing $C=1$ and $L=3$. The leftmost panels are Lyapunov stability diagrams while the other ones are isospike diagrams [16,25], obtained by counting spikes per period of the $x, y$, and $z$ variables, respectively, from left to right. The horizontal lines mark $\alpha=0.6$. White boxes refer to successive magnifications on the leftmost column. Lyapunov diagrams display $1400 \times 1400$ exponents, while other panels display grids of $1000 \times 1000$ parameter points.

more than 19 spikes are plotted by recycling the 19 basic colors modulo 19. Black represents "chaos", i.e. lack of numerically detectable periodicity. Isospike diagrams are also useful to analyze experimental data [33]. The computational cost to obtain isospike diagrams is significantly smaller than the cost to obtain Lyapunov diagrams.

Panels a and b in Figure 7 are Lyapunov diagrams showing the tunable intervals for periodic and chaotic motions for a section of the $\alpha \times L$ plane. The distribution of phases is similar to the ones described before. A new type of stability diagram is shown in Figure 7 where parameters are color-coded according to the period of their corresponding oscillations. Chaotic oscillations are shown in black. As it is clear, period diagrams can also reveal details of the substructures which form periodicity islands. However, to reveal such details in period diagrams it is usually necessary to define an upper cutoff period, namely a fixed value to which a fixed color is attributed whenever a computed period is larger than the cutoff. Without a proper cutoff, the period diagram turns out to be too dark, showing no details. Figures $7 \mathrm{~d}$ and $7 \mathrm{e}$ display isospike diagrams obtained by counting spikes of the $x$ and $y$ variables. As before, spikes of $z$ produce a diagram similar to Figure $7 d$. From panels d and e it is easy to recognize that, although the $x$ and $y$ variables oscillate independently, the overall structure of the diagrams is the same, the difference being simply the number of spikes per period.

Finally, Figure 8 presents period and isospike diagrams similar to the ones in Figure 7 , but for the control planes $\alpha \times C$ and $C \times L$. Once again, although stability 

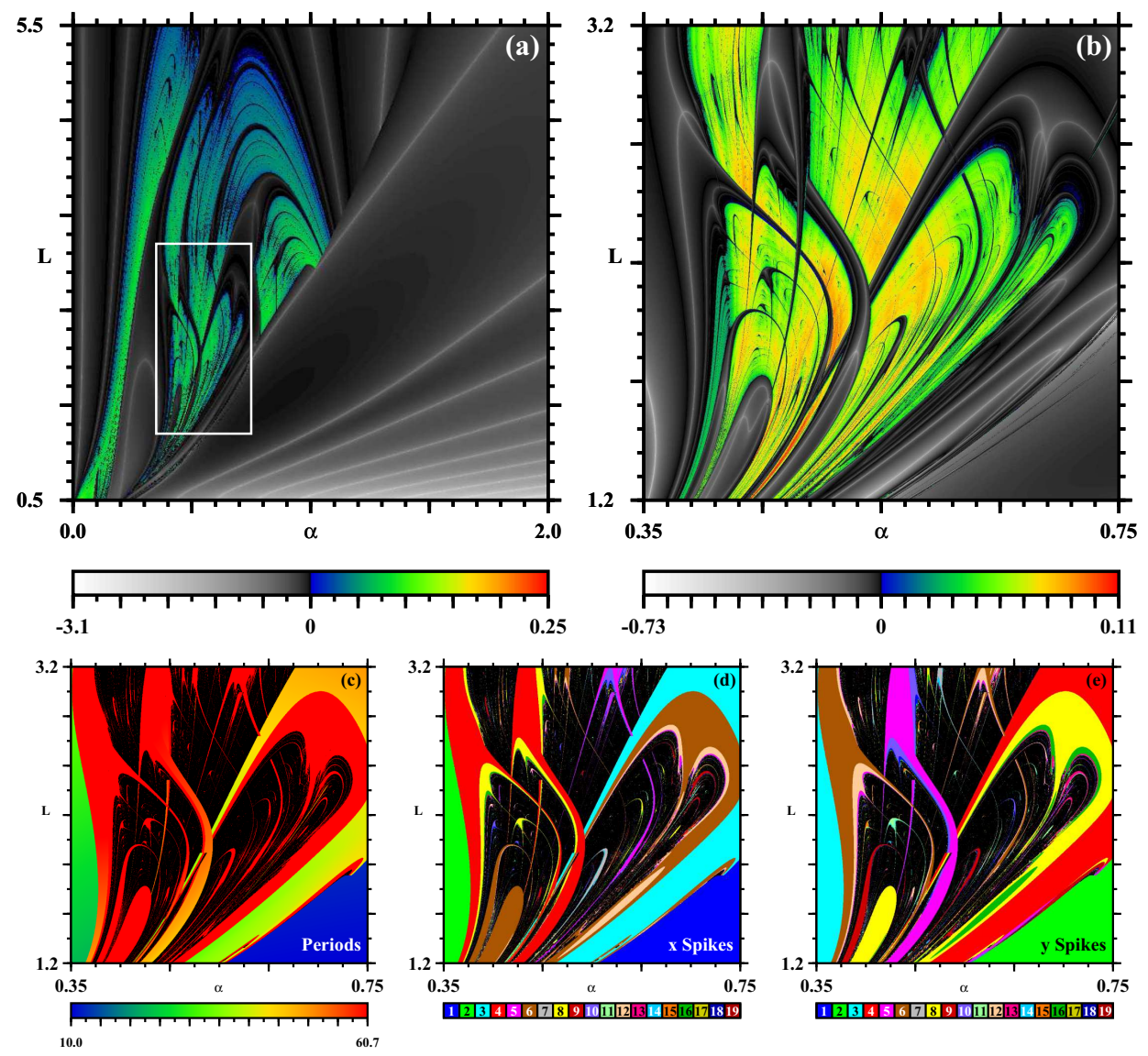

Fig. 7. (a) Extended view of the stability regions in the $\alpha \times L$ control plane. (b) Magnification of the white box in (a) showing details of the chaotic phase. (c) Parameter classification according to the distribution of the oscillation period. Chaos is represented in black. (d) Isospike diagram $[16,25]$ obtained by counting spikes of $x$, which essentially coincide with the spikes of $z$. (e) Isospike diagram obtained by counting spikes of $y$. Here $C=1$ and $\beta=1.5$. Each panel shows a grid of $1000 \times 1000$ points.

regions appear stretched and distorted, there is no indication of periodicity hubs and the associated nesting of spirals. Both control planes display a relatively small region where chaotic oscillations are to be expected. Furthermore, the inner substructure generated by the $y$ oscillations is more complex than the corresponding ones recorded for the $x$ variable.

\section{Conclusions and outlook}

Detailed stability diagrams predicting the location and relative abundance of tunable ranges for periodic and chaotic motions for the simplest possible circuit containing a memristor were computed. This was done for the six possible two-dimensional sections of the control parameter space. The memristor circuit displays a plethora of chaotic phases containing intricate self-organized sequences of windows of stable periodic oscillations. As it is clear from the stability diagrams reported above, changing the control parameters $C$ and $L$, of the linear components, produces effects analogous to changing $\alpha$ and $\beta$, which control the memristor. Our initial expectation of finding 

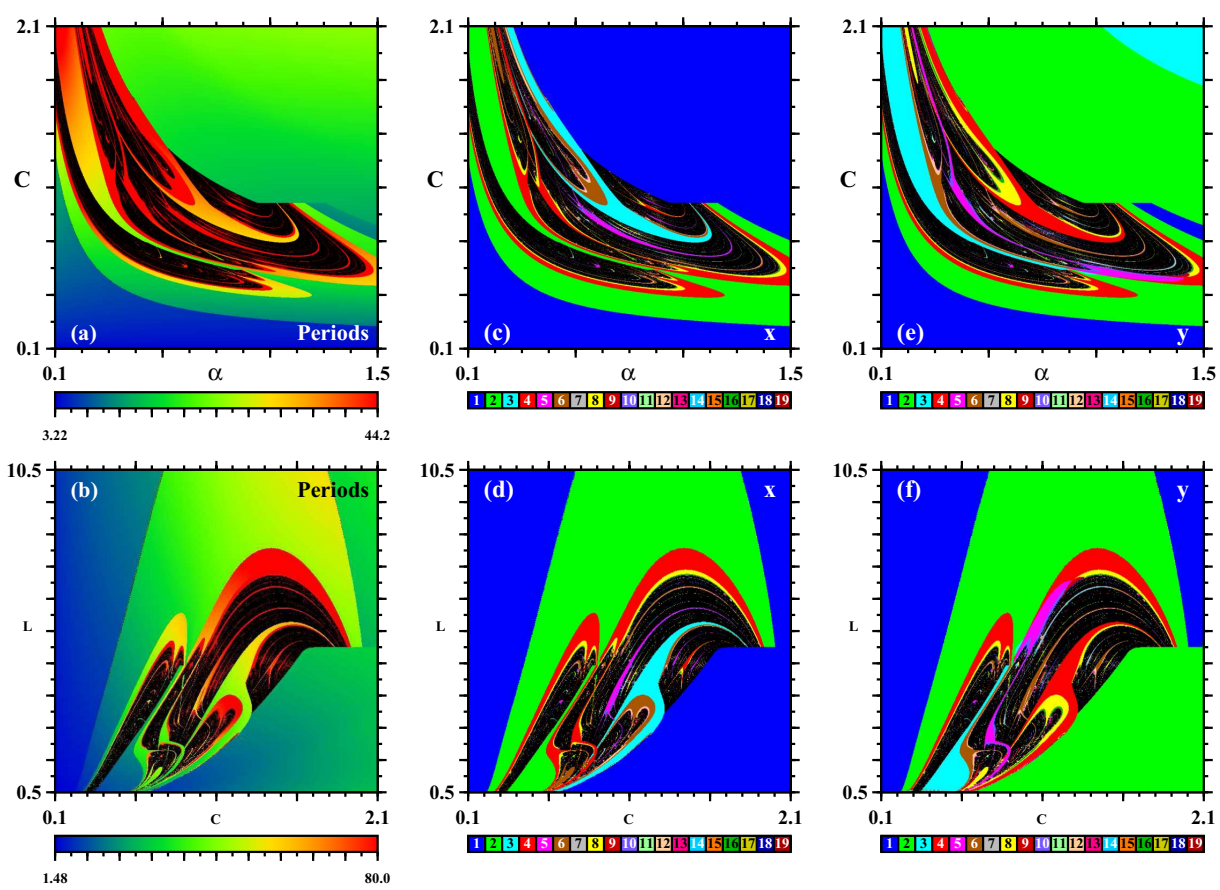

Fig. 8. Stability diagrams showing the relevant control regions for two distinct control planes. As in Figure 7, panels a and b display the periods, while the other panels were obtained by counting spikes per period of $x$ and $y$, respectively, as indicated. Spikes of $z$ essentially coincide with spikes of $x$ and are not shown. Individual panels display grids displaying $1000 \times 1000$ parameter points.

periodicity hubs and spirals [33] in the system was not confirmed, despite the fact that the distribution of oscillations, chaotic or not, shows rather complicated structures and substructures.

Several additional stability diagrams (not shown here) were also computed for specific areas of the control parameters, with some of them apparently hinting to the possible existence of regions with complicated dynamics embedded in the chaotic phase. A search for such behaviors demands considerable additional investment of computer time and, therefore, is not further pursued here. It is hoped that the rich predictions of an organized unfolding of oscillations discovered in the present prospection may motivate their experimental investigation, along the lines recently done for a Duffing-like proxy designed to bypass noisy spectra conspicuously present in oscillators [33], in the standard circuit of Chua [34], and in other scenarios [35,36].

Open Access funding provided by Max-Planck Society. Work done in the framework of an Advanced Study Group on Forecasting with Lyapunov vectors, at the Max-Planck Institute for the Physics of Complex Systems, Dresden. The author was supported by CNPq, Brazil. All stability diagrams were computed at the CESUP-UFRGS supercomputing cluster, in Porto Alegre, Brazil.

Open Access This is an open access article distributed under the terms of the Creative Commons Attribution License (http://creativecommons.org/licenses/by/4.0/), which permits unrestricted use, distribution, and reproduction in any medium, provided the original work is properly cited. 


\section{References}

1. B. Muthuswamy, L.O. Chua, Int. J. Bifurc. Chaos 20, 1567 (2010)

2. S. Vaidyanathan, C. Volos, Advances in memristors, memristive devices and systems (Springer, Cham, 2017)

3. A.G. Radwan, M.E. Fouda, On the mathematical modeling of memristor, memcapacitor, and meminductor (Springer, Cham, 2015)

4. A. Adamatzky, L. Chua, Memristor networks (Springer, Cham, 2014)

5. S. Kumar, J.P. Strachan, R.S. Williams, Nature 548, 318 (2017)

6. S. Kumar, N. Davila, Z. Wang, X. Huang, J.P. Strachan, D. Vine, A.L.D. Kilcoyne, Y. Nishi, R.S. Williams, Nanoscale 9, 1793 (2017)

7. G. Wang, M. Cui, B. Cai, X. Wang, T. Hu, Math. Prob. Eng. 2015, 561901 (2015)

8. A. Ascoli, S. Slesazeck, H. Mahne, R. Tetzlaff, T. Mikolajick, IEEE Trans. Circuits Syst. 62, $1165(2015)$

9. L. Chua, Nanotechnology 24, 383001 (2013)

10. T. Driscoll, Y.V. Pershin, D.N. Basov, M. di Ventra, Appl. Phys. A 102, 885 (2011)

11. J.M. Ginoux, R. Meucci, S. Euzzor, A. di Garbo, Int. J. Bifurc. Chaos 28, 1850128 (2018)

12. J.M. Ginoux, C. Letellier, L.O. Chua, Int. J. Bifurc. Chaos 20, 3819 (2010)

13. C. Bonatto, J.A.C. Gallas, Phys. Rev. Lett. 101, 054101 (2008)

14. J.A.C. Gallas, Int. J. Bifurc. Chaos 20, 197 (2010)

15. R. Vitolo, P. Glendinning, J.A.C. Gallas, Phys. Rev. E 84, 016216 (2011)

16. J.A.C. Gallas, Adv. At. Mol. Opt. Phys. 65, 127 (2016)

17. G.M. Ramírez-Ávila, J.A.C. Gallas, Phys. Lett. A 375, 143 (2010)

18. Y. Zhang, X. Zhang, Int. J. Bifurc. Chaos 23, 1350136 (2013)

19. J. Llibre, C. Valls, J. Nonlinear Math. Phys. 19, 1250029 (2012)

20. F. Yuan, G. Wang, X. Wang, Chaos 27, 033103 (2017)

21. H. Wu, B. Bao, Z. Liu, Q. Xu, P. Jiang, Nonlinear Dyn. 83, 893 (2016)

22. L.V. Gambuzza, L. Fortuna, M. Frasca, E. Gale, Int. J. Bifurc. Chaos 25, 1550101 (2015)

23. A. Buscarino, L. Fortuna, M. Frasca, L.V. Gambuzza, Int. J. Bifurc. Chaos 23, 1330015 (2013)

24. B. Muthuswamy, Int. J. Bifurc. Chaos 20, 1353 (2010)

25. J.G. Freire, J.A.C. Gallas, Phys. Chem. Chem. Phys. 13, 12191 (2011)

26. J.A.C. Gallas, Phys. Rev. Lett. 70, 2714 (1993)

27. E.N. Lorenz, Physica D 237, 1689 (2008)

28. W. Façanha, B. Oldeman, L. Glass, Phys. Lett. A 377, 1264 (2013)

29. R.E. Francke, T. Pöschel, J.A.C. Gallas, Phys. Rev. E 87, 042907 (2013)

30. L. Junges, J.A.C. Gallas, Phys. Lett. A 376, 2109 (2012)

31. J. Bragard, H. Pleiner, O.J. Suarez, P. Vargas, J.A.C. Gallas, D. Laroze, Phys. Rev. E 84, $037202(2011)$

32. M.R. Gallas, M.R. Gallas, J.A.C. Gallas, Eur. Phys. J. Special Topics 223, 2131 (2014)

33. A. Sack, J.G. Freire, E. Lindberg, T. Pöschel, J.A.C. Gallas, Sci. Rep. 3, 3350 (2013)

34. F.F.G. Sousa, R.M. Rubinger, J.C. Sartorelli, H.A. Albuquerque, M.S. Baptista, Chaos 26, $083107(2016)$

35. D.W.C. Marcondes, G.F. Comassetto, B.G. Pedro, J.C.C. Vieira, A. Hoff, F. Prebianca, C. Manchein, H.A. Albuquerque, Int. J. Bifurc. Chaos 27, 1750175 (2017)

36. C. Manchein, H.A. Albuquerque, L.F. Mello, Int. J. Bifurc. Chaos 28, 1830038 (2018) 